\title{
Cytohesin-1 controls the activation of RhoA and modulates integrin-dependent adhesion and migration of dendritic cells
}

\author{
*Thomas Quast, ${ }^{1}$ *Barbara Tappertzhofen, ${ }^{1}$ Cora Schild, ${ }^{1}$ Jessica Grell, ${ }^{1}$ Niklas Czeloth, ${ }^{2}$ Reinhold Förster, ${ }^{2}$ Ronen Alon, ${ }^{3}$ \\ Line Fraemohs, ${ }^{4}$ Katrin Dreck, ${ }^{1}$ Christian Weber, ${ }^{4}$ Tim Lämmermann, ${ }^{5}$ Michael Sixt, ${ }^{5}$ and Waldemar Kolanus ${ }^{1}$ \\ ${ }^{1}$ Life and Medical Sciences (LIMES) Institute, Laboratory for Molecular Immunology, University of Bonn, Bonn, Germany; ${ }^{2}$ Institute of Immunology, Hannover \\ Medical School, Hannover, Germany; ${ }^{3}$ Department of Immunology, The Weizmann Institute of Science, Rehovot, Israel; ${ }^{4}$ Institute for Molecular Cardiovascular \\ Research (IMCAR), Rheinisch-Westfaelische Technische Hochschule (RWTH) University Aachen, Aachen, Germany; and ${ }^{5}$ Max-Planck-Institute for \\ Biochemistry, Martinsried, Germany
}

\begin{abstract}
Adhesion and motility of mammalian leukocytes are essential requirements for innate and adaptive immune defense mechanisms. We show here that the guanine nucleotide exchange factor cytohesin-1, which had previously been demonstrated to be an important component of beta-2 integrin activation in lymphocytes, regulates the activation of the small GTPase RhoA in primary dendritic
\end{abstract}

cells (DCs). Cytohesin-1 and RhoA are both required for the induction of chemokinedependent conformational changes of the integrin beta-2 subunit of DCs during adhesion under physiological flow conditions. Furthermore, use of RNAi in murine bone marrow DCs (BM-DCs) revealed that interference with cytohesin-1 signaling impairs migration of wild-type dendritic cells in complex 3D environments and in vivo. This phenotype was not observed in the complete absence of integrins. We thus demonstrate an essential role of cytohesin-1/RhoA during ameboid migration in the presence of integrins and further suggest that DCs without integrins switch to a different migration mode. (Blood. 2009;113:5801-5810)

\section{Introduction}

Integrin-mediated adhesion is an essential function of most metazoan cells, and plays a key role in, for example, organ development, the maintenance of tissue architecture, blood clotting, and many aspects of leukocyte biology. The mammalian integrin family consists of 24 heterodimeric cell surface receptors that bear distinct functional binding specificities for extracellular matrix proteins and plasma membrane counterreceptors. ${ }^{1}$ Immune cells have highly specific requirements for timing and location of cell-cell and -matrix contacts, which need to be finely tuned to variable conditions of homeostasis and inflammation.

An important hallmark of integrin function in the immune system is the rapid adhesion of leukocytes to activated endothelia, a process that is essential for immune cell egress from the vasculature into either lymphoid organs or into infected tissues. To perform such functions, integrins on leukocytes have to be activated by a process that has been termed "inside-out" signaling. It is now generally believed that integrins on circulating, resting leukocytes are conformationally restricted, so that they normally cannot bind to their protein ligands, such as ICAM-1 or VCAM-1. However, if a leukocyte becomes tethered to an endothelial cell, for example, by selectin-mediated adhesive "rolling," immune cell chemokine receptors such as CCR7 or CXCR4 may be activated by chemokines present on the activated vessel wall. ${ }^{2,3}$ This, in turn, results in intracellular, heterotrimeric G-protein-coupled signal transduction, which enables very fast conformational changes and ligand binding of cell surface integrins, resulting in a robust firm adhesion, the so-called integrin-mediated leukocyte "arrest." 3 Shear forces imposed by the blood flow apparently play an important role in the regulation of this process. ${ }^{4}$ The mode of activation of immune cell integrins is incompletely understood. Several intracellular proteins that directly or indirectly interact with the cytoplasmic domains of the leukocyte-specific beta-2 integrins, such as LFA-1, have been implicated in this function. These include the actin- and integrinbinding protein talin, which is important for the general coupling of integrin-mediated cell adhesion to the actin cytoskeleton, ${ }^{5-7}$ the GTPase Rap $1^{7-9}$ and its effector proteins RAPL and RIAM, ${ }^{9-11}$ the ADAP/Vav/SLP76 complex in T cells, ${ }^{12-14}$ the Rho GTPases $\mathrm{RhoA}^{15}$ and $\mathrm{CdC} 42,{ }^{16}$ and, recently, the so-called kindlin proteins. ${ }^{17,18}$

Our laboratory has identified the guanine nucleotide exchange factor (GEF) for ADP-ribosylation factor (ARF) GTPases, cytohesin-1, as an LFA-1 interacting protein. Cytohesin-1 binds specifically to the cytoplasmic portion of the integrin beta- 2 subunit CD18. ${ }^{19}$ In Jurkat T cells, overexpression of cytohesin-1 results in up-regulation of LFA-1 adhesion to ICAM-1, whereas the expression of the isolated $\mathrm{PH}$ domain of cytohesin-1, which is required for inositol-phospholipid-mediated membrane recruitment of the protein, abrogates LFA-1 activation. ${ }^{20,21}$ Cytohesin-1 was also found to be an important regulator of beta- 2 integrinmediated recruitment of monocytic cells to human endothelial cells under physiological flow conditions..$^{22}$ The precise mechanism of integrin activation by cytohesin- 1 is not understood but it involves both direct interactions, possibly resulting in triggering conformational switches, and signal transduction through its GEF domain, which activates ARF GTPases, a function that is important for cell spreading. ${ }^{23}$
Submitted August 26, 2008; accepted March 27, 2009. Prepublished online as Blood First Edition paper, April 3, 2009; DOI 10.1182/blood-2008-08-176123.

${ }^{*}$ T.Q. and B.T. contributed equally to this work.

The online version of this article contains a data supplement.
The publication costs of this article were defrayed in part by page charge payment. Therefore, and solely to indicate this fact, this article is hereby marked "advertisement" in accordance with 18 USC section 1734. 
Another important requirement for immune cell functionality is their ability to rapidly move through complex environments in the interstitium. So-called dendritic cells (DCs) of myeloid origin become activated by pathogen encounter in infected tissues through Toll-like receptor and inflammatory cytokine signaling. During this process, DCs "mature," that is, they undergo massive changes in morphology that enable their rapid motility. At the same time, DCs up-regulate surface proteins that enable their trafficking to lymphoid organs, for example, chemokine receptors such as CCR7, as well as costimulatory receptors and $\mathrm{MHC}$ molecules that are required for proper T-cell activation by DCs in secondary lymphoid organs. The importance of integrins in coupling ECM recognition to cytoskeletal rearrangements in interstitial immune cell migration has been subject to debate. Several years ago, seminal work by Friedl et $\mathrm{al}^{24}$ suggested that integrins might not be required for interstitial motility of $\mathrm{T}$ cells. Recently, an elegant study published by Lämmermann et $\mathrm{al}^{25}$ which used dendritic cells and neutrophil granulocytes from mice that lack all integrins on the leukocyte surface, showed that integrin-deficient immune cells apparently migrate normally in vitro and in vivo. However, rapid interstitial migration of these cells was shown to be dependent on rapid forward flow of actin at the cell front, and on their capacity to deform organelles such as the nucleus to "squeeze" through narrow passages in collagen/ matrix webs. This latter ability was dependent on intracellular signaling pathways controlled by the GTPase RhoA. ${ }^{25}$

We show here that the ARF-GEF and beta- 2 integrin binding protein cytohesin-1 serves a novel, dual role in mediating dendritic cell adhesion and migration, and the common denominator of both functions is the positioning of the protein upstream of RhoA. The expression of cytohesin-1 protein has been efficiently silenced by RNAi in mature dendritic cells of both human and murine origin. Interestingly, mature DCs are capable of activating their beta- 2 integrins by chemokine signaling under physiological flow conditions, a function that is linked to PI3-kinase and cytohesin-1mediated inside-out signal transduction. Furthermore, cytohesin-1 is shown for the first time to control DC migration in 3D collagen gels, and to regulate mature DC migration in vivo by functioning upstream of RhoA activation. Strikingly, cytohesin-1 is apparently not required for 3D migration of integrin knockout DCs (quadruple ablation of the integrin alpha-v, beta-1, beta-2, and beta-7 genes, respectively ${ }^{25}$ ). These findings suggest that immune cell migration may also be subclassified into distinct modes, and that the integrin-dependent mode selectively requires the function of cytohesin-1 upstream of RhoA.

\section{Methods}

\section{Generation of human monocyte-derived DCs}

See supplemental methods (available on the Blood website; see the Supplemental Materials link at the top of the online article). Animal care and experiments were done in compliance with institutional guidelines and the German law for Welfare of Laboratory Animals. Isolation of primary cells was approved by the University of Bonn ethics committee.

\section{Generation of murine bone marrow-derived DCs}

See supplemental methods.

\section{Generation of integrin-deficient bone marrow-derived DCs}

Generation of integrin alpha-v-, beta-1-, beta-2-, and beta-7-deficient bone marrow-derived DCs (BM-DCs) has been described by Lämmermann et al. ${ }^{25}$

\section{Antibodies}

The following monoclonal antibodies were used against the human antigens: HLA-DR, CD80, CD86 (all from BD Pharmingen, San Diego, CA), anti-CCR7 and anti-CXCR4 (both from R\&D Systems, Minneapolis, MN), anti-CD18 MHM23 (Dako, Carpinteria, CA). The 327C ${ }^{26,27}$ specific for activated human CD18 epitopes was a gift from D. Staunton (Seattle, WA). E. Kremmer (Munich, Germany) generated a rat monoclonal antibody (7H2) against human cytohesin-1 as previously described ${ }^{23}$ and 2D7, which also detects cytohesin. These monoclonal antibodies were against the following mouse antigens: MHCII, CD80 (clone 16-10A1), CD86 (clone B7-2; all from eBiosciences, San Diego, CA). These monoclonal antibodies were against both human and mouse antigens: anti-RhoA (Santa Cruz Biotechnology, Santa Cruz, CA) and anti-Rac (Chemicon, Temecula, CA). For flow cytometry analysis, the following isotype-matched antibody controls were used: rat IgGI PE (BD Pharmingen) and rat IgGI FITC (clone eBRG1; eBiosciences).

\section{Oligonucleotide sequences and plasmids}

See supplemental methods.

\section{siRNA transfections}

Oligonucleotides (5 $\mu \mathrm{g}$ siRNA against the target sequence of cytohesin-1; $10 \mu \mathrm{g}$ against the target sequence of RhoA or Rac1, respectively) were transferred to a 4-mm cuvette (Bridge, Providence, RI) and incubated for 3 minutes with $4 \times 10^{6}$ immature DCs (imDCs) in $100 \mu \mathrm{L}$ Optimem (Invitrogen, Frederick, MD) before electroporation in a Gene Pulser X cell + CE module (Bio-Rad, Hercules, CA). Pulse conditions were square wave, $1000 \mathrm{~V}, 2$ pulses, and 0.5-ms pulse length. Forty-eight hours after electroporation, imDCs were stimulated to mature (Document S1). Seventytwo to 96 hours after electroporation, RNAi efficiency was tested using a standard Western blot protocol and silenced mature DCs (mDCs) were used for functional assays.

\section{Transfection of monocyte-derived DCs with plasmid DNA}

See supplemental methods.

\section{Confocal laser scanning microscopy}

See supplemental methods.

\section{Static adhesion assays}

See supplemental methods.

Static adhesion assay with GFP-transfected monocyte-derived DCs

See supplemental methods.

\section{Laminar flow assays}

See supplemental methods.

\section{BM-DC/T-cell adhesion}

See supplemental methods.

\section{Transwell migration assay}

See supplemental methods.

\section{Migration assay with GFP-transfected monocyte-derived dendritic cells}

See supplemental methods.

\section{In vivo migration assays}

See supplemental methods. 


\section{D collagen gel chemotaxis assay}

$3 \mathrm{D}$ chemotaxis assays were processed as described by Friedl et a ${ }^{24}$ with the following modifications. Collagen I (Pure Col; Inamed, Fremont, CA) was mixed with $0.7 \%$ sodium bicarbonate (Invitrogen) and $1 \times$ MEM (SigmaAldrich, St Louis, MO) on ice. BM-DCs in VLE-RPMI/0.5\% FCS were carefully mixed with the collagen solution at a 2:1 ratio, resulting in gels with a collagen concentration of $1.6 \mathrm{mg} / \mathrm{mL}$ and a final cell concentration of $1.5 \times 10^{6}$ cells $/ \mathrm{mL}$ gel. Collagen-BM-DC mixtures were placed in custommade chemotaxis chambers and incubated at $37^{\circ} \mathrm{C} / 5 \% \mathrm{CO}_{2}$ for 90 minutes. After collagen polymerization, $600 \mathrm{ng} / \mathrm{mL}$ CCL19 (R\&D Systems) in VLE-RPMI/0.5\% FCS was added on top of the gel. Time-lapse series of motile BM-DCs in 3D collagen gel were recorded using a fully automated inverted TE Eclipse microscope (Nikon, Tokyo, Japan), equipped with a $10 \times$ phase-contrast objective (Nikon), motorized xyz-stage (Märzhäuser, Wetzlar, Germany), a climate chamber $\left(37^{\circ} \mathrm{C} / 5 \% \mathrm{CO}_{2}\right.$ humidity), and a CCD-1300 camera (Vosskühler, Osnabrücu, Germany). For every single experiment 60 individual BM-DCs were tracked over 3 hours (5 minutes/ frame) using the manual tracking plug-in of ImageJ (National Institutes of Health [NIH], Bethesda, MD). Subsequently, cell directionality, forward migration index, and velocity were calculated and visualized as plots and videos by analyzing the acquired data with the Chemotaxis and Migration tool plug-in (Ibidi, Martinsried, Germany) for ImageJ.

\section{GTPase activation assays}

See supplemental methods.

\section{Statistical analysis}

Statistical significance between data groups was determined by analysis of variance (ANOVA) with the Instat software (GraphPad, San Diego, CA) and considered to be significantly different at $P$ values less than .05. The $P$ values are depicted as asterisks in the figures. *** indicates $P$ value less than $.001 ; * *, P$ value less than .01 ; and $*, P$ value less than .05 .

\section{Results}

\section{Efficient RNAi of cytohesin-1 does not affect maturation of} human or murine dendritic cells

The mRNA of cytohesin-1 is up-regulated in human monocytederived dendritic cells (Mo-DCs) upon maturation induced by lipopolysaccharide (LPS) and TNF- $\alpha .{ }^{26}$ We performed Western blot analysis to establish the up-regulation on the protein level (Figure 1A). On day 7 after isolation of monocytes, expression of cytohesin-1 was detectable in immature dendritic cells by the specific anti-cytohesin-1 antibody $7 \mathrm{H} 2$. Incubation of the cells with LPS and TNF- $\alpha$ yielded a strong increase of cytohesin-1 expression, which reaches peak levels at day 9. The maturation of DCs was determined by controlling the expression of the characteristic markers HLA-DR, CD80, and CD86 by fluorescenceactivated cell sorting (FACS) analysis.

RNA interference can be used to specifically inhibit gene expression and to analyze gene function. ${ }^{27}$ Using electroporation, we silenced cytohesin-1 protein expression during maturation of DCs. Compared with Mo-DCs that were transfected with a control siRNA, RNAi of cytohesin-1 dramatically reduced specific protein expression, as detected by Western blot analysis (Figure 1B). In contrast to human Mo-DCs, cytohesin-1 is constitutively expressed in murine BM-DCs (not shown). Nonetheless, RNAi-mediated silencing of cytohesin-1 yielded a similar reduction of specific protein expression in this cell type (Figure 1C).

To test whether RNAi of cytohesin-1 interferes with maturation of DCs, we measured the cell surface expression of maturation markers with the help of flow cytometry (FACS). Figure S9A
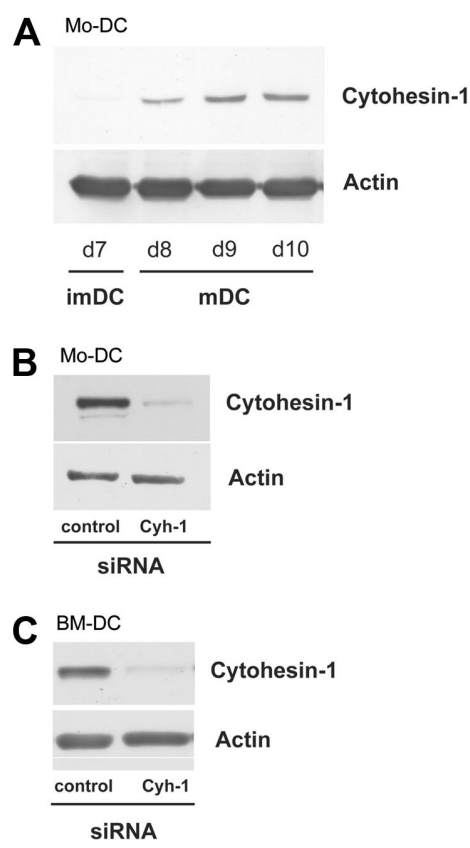

Figure 1. Cytohesin-1 is up-regulated in human Mo-DCs during maturation, and is efficiently silenced by RNA i in both human and murine DCs. Western blot analysis shows that cytohesin-1 expression is up-regulated in human monocytederived dendritic cells (Mo-DCs) upon maturation induced by LPS $(1 \mu \mathrm{g} / \mathrm{mL}) / T N F$ alpha $(50 \mathrm{ng} / \mathrm{mL}$; panel $A$ lanes d8, d9, d10). Expression of cytohesin- 1 is barely detectable in immature dendritic cells (imDCs), but maturation results in a strong increase of cytohesin-1 expression, reaching maximum levels at day $9(A)$. Using square wave electroporation, cytohesin-1 expression was efficiently silenced in mature DCs. Western blot analysis shows that RNAi of cytohesin-1 reduces specific protein expression 96 hours after electroporation in human Mo-DCs (B) or murine bone marrow-derived DCs (BM-DCs, C).

shows that maturation of Mo-DCs is not altered after transfection with a cytohesin-1-specific siRNA, compared with control cells. Furthermore, we assessed potential effects of cytohesin-1 knockdown on the expression of CCR7 and CXCR4 on mature Mo-DCs. CCR7 and CXCR4 are receptors for the chemokines CCL19 and CXCL12, respectively, which are important mediators of mDC migration in vivo. As shown in Figure S9A, RNAi of cytohesin-1 interfere neither with the expression of CCR7, nor with that of CXCR4. Hence, the reduction of cytohesin-1 protein expression does not affect the cellular levels of a variety of important surface proteins of mature Mo-DCs. Consistently, RNAi of cytohesin-1 does not significantly affect maturation marker expression of murine BM-DCs, compared with control samples (Figure S9B).

\section{Cytohesin-1 mediates chemokine-induced activation of CD18 in mature Mo-DCs}

We have previously shown that overexpressed cytohesin-1 colocalizes with CD18 in Jurkat cells and activates LFA-1 adhesion to ICAM-1. ${ }^{23}$ Here, we demonstrate that endogenous cytohesin-1 colocalizes with the beta- 2 integrin subunit CD18 in mature Mo-DCs upon chemokine stimulation (Figure 2A). Immunofluorescence tracings reveal that endogenous cytohesin-1 is predominantly located in the cytoplasm in Mo-DCs. However, stimulation of mature Mo-DCs with the chemokine CCL19 induces translocation of cytohesin-1 to the plasma membrane (Figure 2A middle panel) where it remains colocalized with CD18 after antibodymediated clustering of the integrin (Figure 2A bottom panel). To elucidate the functional significance of this finding, we analyzed 

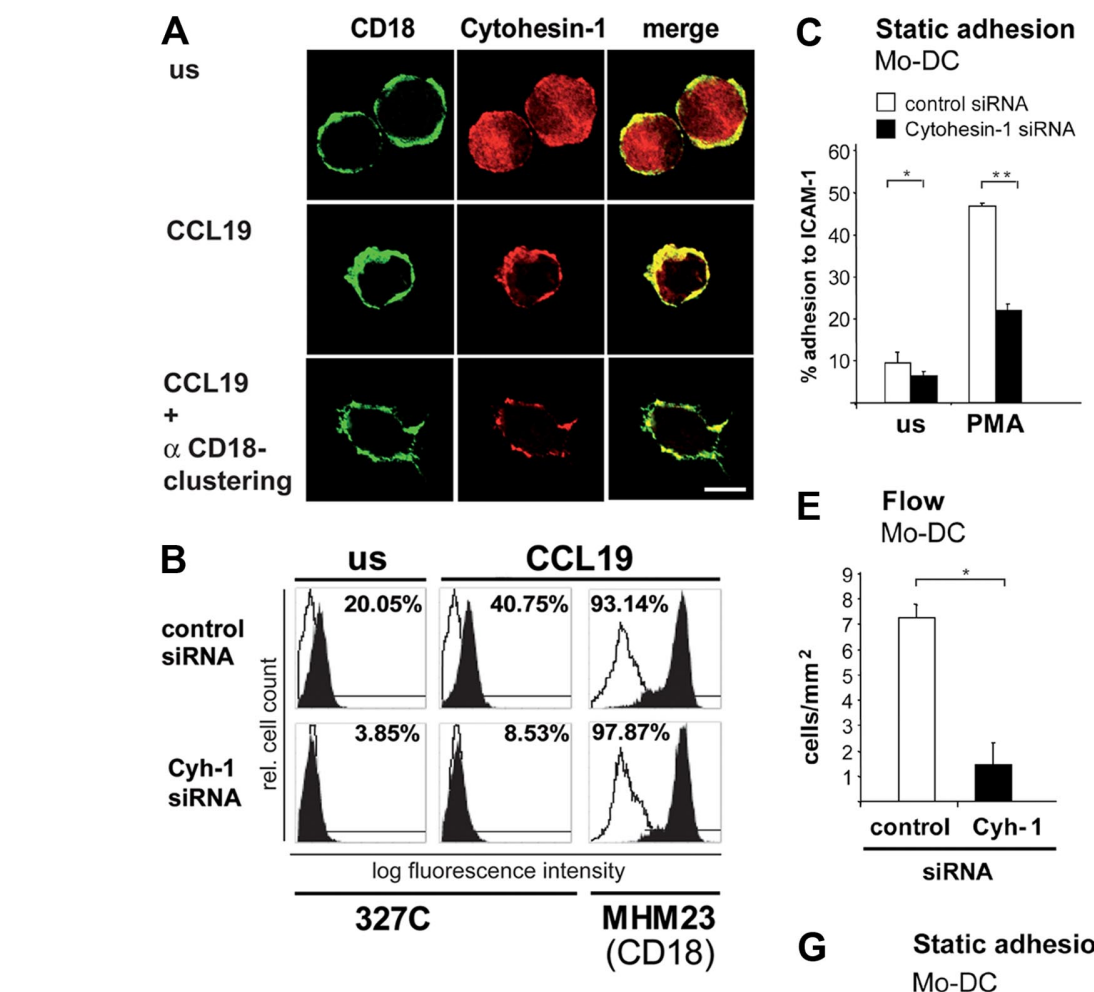

D BM-DC - $\mathrm{T}$ cell adhesion
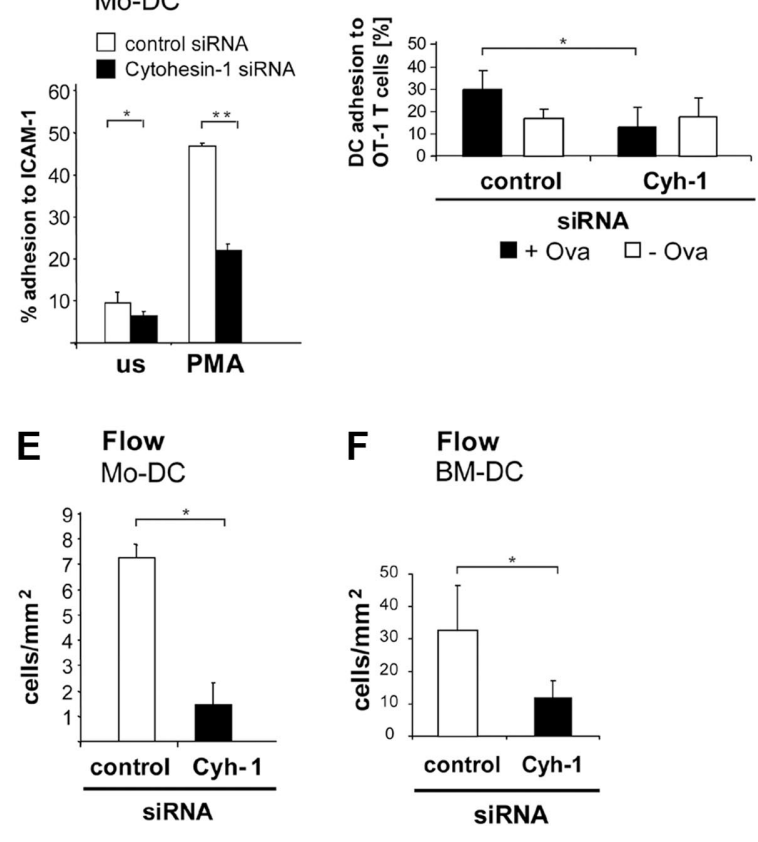

\section{G Static adhesion Mo-DC}

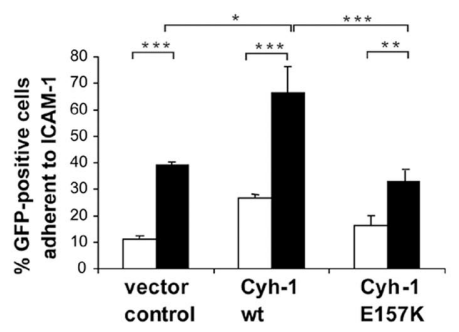

- PMA

$\square$ unstimulated

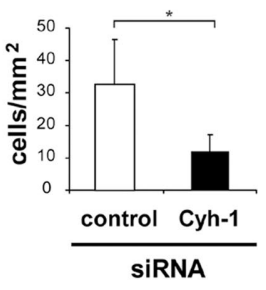

Flow

BM-DC

Figure 2. Cytohesin-1 (red fluorescence) colocalizes with CD18 (green fluorescence) and mediates both CD18 activation and adhesion. In contrast to unstimulated mature Mo-DCs where cytohesin-1 (detected by mAb 7H2) localizes to the cytoplasm (A top panel), stimulation with $200 \mathrm{ng} / \mathrm{mL} \mathrm{CCL} 19$ for 10 minutes induces translocation of cytohesin-1 to the plasma membrane (A middle panel) where it colocalizes with beta- 2 integrins, clustered by an anti-CD18 antibody (A bottom panel; bar represents $5 \mu \mathrm{m}$ ). All shown fluorescence staining images are confocal images, focused on the medial z-position of the respective cells, that is, the cytoplasm and the plasma membrane (A). The different fluorochromes of secondary antibodies in the costaining of cytohesin-1 and CD18 were acquired sequentially by the use of a confocal laser scanning microscope (A). Flow cytometric analysis shows that CCL19 (200 ng/mL for 10 minutes) strongly increases the expression of the high-affinity state (detected by mAb $327 \mathrm{C}$ ) of LFA-1 (B). RNAi of cytohesin-1 reduces expression of the activation epitope, compared with control cells (B). In contrast, a constitutive CD18 epitope (detected by mAb MHM23) is not altered after RNAi of cytohesin-1 (B). The empty profiles in the flow cytometry histograms indicate the background cell stainings by isotype controls (B). RNAi of cytohesin-1 strongly reduces static adhesion of Mo-DCs to ICAM-1-Fc compared with control cells (C). Cytohesin-1 siRNAs selectively down-regulate antigen-specific BM-DC adhesion to OT-1 T cells (D). Under flow conditions, RNAi of cytohesin-1 reduces dynamic adhesion of Mo-DCs to human umbilical vein endothelial cells (HUVECs; E) or of BM-DCs to brain endothelioma cells ( $B$ end5; F). Overexpression of wild-type cytohesin-1 but not of the GEF-deficient E157K mutant increases static adhesion of GFP-cotransfected Mo-DCs to ICAM-1-Fc (G). In the static adhesion assays, Mo-DCs were stimulated with $50 \mathrm{ng} / \mathrm{mL}$ PMA for 60 minutes at $37^{\circ} \mathrm{C}(\mathrm{C}, \mathrm{G})$. Error bars indicate $\pm \mathrm{SD}(\mathrm{C}-\mathrm{G})$. ${ }^{* \star *} P<.001$, ${ }^{* *} P<.01,{ }^{*} P<.05$. Each experiment was repeated at least 3 times independently. Each single experiment was performed in duplicate.

the affinity state of beta-2 integrins on mature Mo-DCs after stimulation with CCL19. Chemokines induce a rapid affinity increase of LFA-1, which is sufficient to cause an arrest of circulating lymphocytes on endothelial cells. ${ }^{28}$ Integrin activation is accompanied by conformational changes. The antibody $327 \mathrm{C}$ may be used to report CD18 epitopes that are expressed only on activated beta- 2 integrins because $327 \mathrm{C}$ detects the high-affinity state of LFA-1. ${ }^{29,30}$ Using this antibody, we performed FACS staining of unstimulated and CCL19-stimulated mature Mo-DCs (Figure 2B). Unstimulated Mo-DCs showed partial activation of $\mathrm{CD} 18$, the level of which differed between cell donors (data not shown). However, CCL19 strongly increased the expression of the $327 \mathrm{C}$ epitope in Mo-DCs, whereas RNAi of cytohesin-1 completely abrogates expression of this epitope (Figure 2B). To test whether RNAi of cytohesin-1 reduces surface expression of CD18, we also analyzed steady-state beta- 2 integrin expression on Mo-
DCs, using antibody MHM23, which recognizes a constitutive CD18 epitope. CD18 expression is not altered after RNAi of cytohesin-1, compared with control cells (Figure 2B). Therefore, cytohesin-1 is an important positive regulator of CD18 affinity in mature Mo-DCs and mediates chemokine-induced inside-out activation of beta- 2 integrins on this cell type.

\section{Cytohesin-1 regulates adhesion of human and murine dendritic} cells

We then assessed static cell adhesion to ICAM-1 using cytohesin-1 knockdown Mo-DCs and control cells. Our results clearly establish cytohesin-1 as an endogenous regulator of beta-2 integrinmediated adhesion, because RNAi of cytohesin-1 markedly reduced static adhesion of unstimulated and PMA-stimulated MoDCs to ICAM-1 (Figure 2C). To test whether cytohesin-1 regulates 
DC adhesion in more physiological settings, we analyzed antigenspecific adhesion of BM-DCs to transgenic OT-1 T cells, which specifically recognize an epitope of the ovalbumin protein. Figure $2 \mathrm{D}$ shows that ovalbumin-loaded BM-DCs specifically adhered to immobilized OT-1 T cells. Importantly, this antigen-specific cellcell interaction is abrogated if cytohesin-1 expression is selectively silenced in the dendritic cell, suggesting that beta-2 integrin activation on DCs contributes to DC/T-cell interactions.

Furthermore, we analyzed the interactions of Mo-DCs and BM-DCs with endothelial cells under physiological flow conditions. To this end, we first tested dynamic adhesion of mature dendritic cells to human umbilical vein endothelial cells (HUVECs) with the help of time-lapse video imaging using a flow chamber. HUVECs were stimulated to express chemokines and adhesion molecules by incubation with TNF- $\alpha$ overnight. In addition, CXCL12 was added, which binds to the surface of endothelial cells. Mature DCs were then injected into the chamber at a steady flow rate of $0.2 \mathrm{~mL} /$ minute $\left(1 \mathrm{dyne} / \mathrm{cm}^{2}\right)$ and subsequent $\mathrm{DC}$ interactions with HUVECs were evaluated in 6 different optical fields within 3 minutes. Adhesion to unstimulated HUVEC monolayers under flow was not observed (not shown). RNAi of cytohesin-1 clearly reduced adhesion of mDCs to HUVECs under flow compared with control cells (Figure 2E). The importance of cytohesin-1 for the activation of DC integrins under flow conditions was also confirmed for the murine system, by assessing BM-DC adhesion to TNF-alpha-activated murine brain endothelioma cells (Figure 2F). All these data are in accordance with our earlier observation, which revealed that cytohesin-1 overexpression enhanced LFA-1-mediated arrest of leukocytes on activated endothelium under flow. ${ }^{22}$ Finally, we assessed whether the GEF function of cytohesin-1 is important for regulating adhesive events of DCs. To this end, we used the novel microporation method "Transfection of monocyte-derived DCs with plasmid DNA" to overexpress wild-type cytohesin-1 or the GEF-deficient mutant E $157 \mathrm{~K}^{23}$ together with a cotransfected plasmid that directs expression of the green fluorescent protein. Figure $2 \mathrm{G}$ shows that overexpression of wild-type cytohesin-1, but not of the E157K mutant, strongly enhances GFP-positive Mo-DC adhesion to ICAM-1.

Taken together, we show strong and coherent evidence that cytohesin-1 - which requires an active GEF function-is an important regulator of integrin-mediated adhesion of dendritic cells in several systems.

\section{DC migration strongly depends on cytohesin-1 function in vitro and in vivo}

An important hallmark of mature DCs is their ability to migrate rapidly and directionally in chemokine gradients. To assess the chemotaxis of Mo-DCs, we first performed so-called transwell migration assays. To this end, transwell polycarbonate filters were either used uncoated, or were coated with the integrin ligands ICAM-1-Fc, fibronectin, or collagen I, respectively. RNAi of cytohesin-1 strongly reduced CCL19- or CXCL12- (not shown) stimulated migration of Mo-DCs in all conditions tested (Figure 3A-D). Overexpression analysis revealed that the GEF function of the protein is also required for this function (Figure 3E). Thus, a GEF-competent cytohesin-1 strongly regulates migration of MoDCs in vitro.

We investigated whether cytohesin-1 is important for migration of mature DCs in vivo. Using RNAi we reduced the expression of cytohesin-1 in mature, murine bone marrow-derived DCs (BMDCs) to approximately $10 \%$ of endogenous levels (Figure 1C).
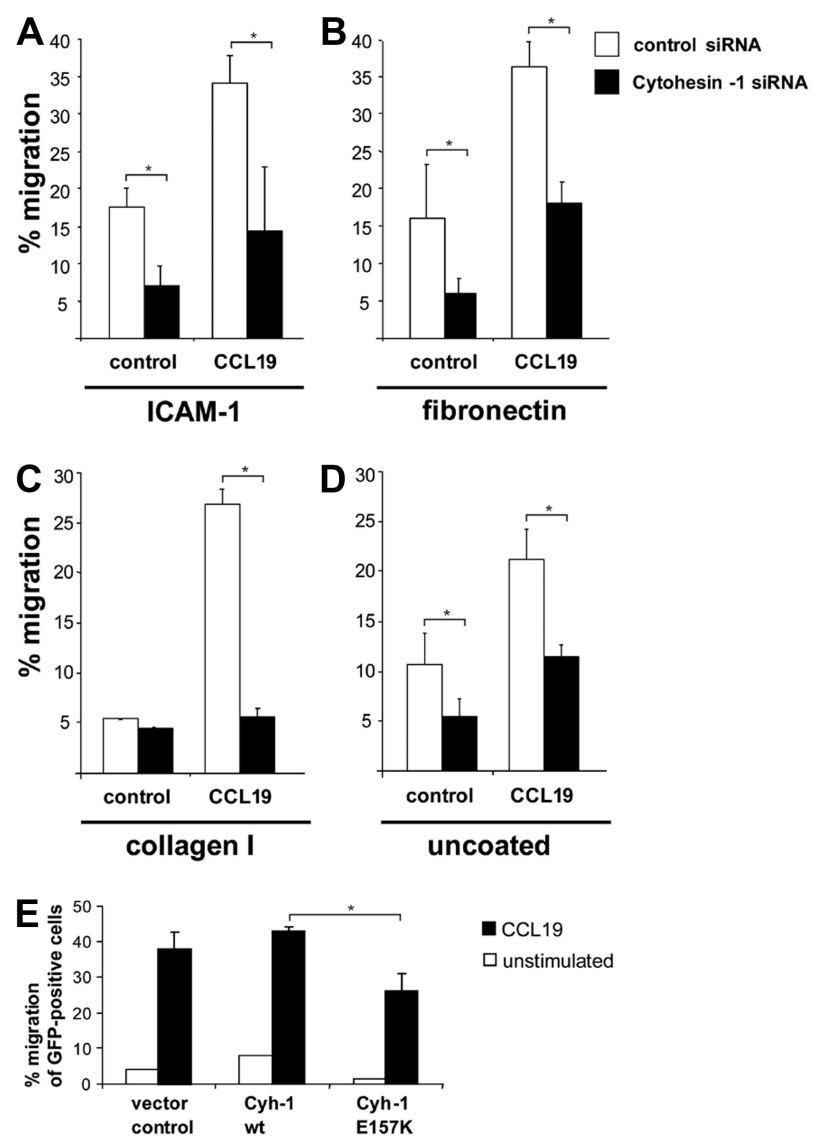

- CCL19

$\square$ unstimulated

Figure 3. RNAi of cytohesin-1 reduces chemotaxis of mature Mo-DCs. Transwell migration assays show that CCL19 $(200 \mathrm{ng} / \mathrm{mL})$-induced chemotaxis is overall strongly reduced in cytohesin-1 knockdown mature Mo-DCs, using integrin ligands ICAM-1-FC ( $10 \mu \mathrm{g} / \mathrm{mL}$; A), fibronectin $(50 \mu \mathrm{g} / \mathrm{mL}$; B), collagen I $(30 \mu \mathrm{g} / \mathrm{mL}$; C), or uncoated filters (D). Overexpression of wild-type cytohesin-1 but not of the GEFdeficient E157K mutant increases chemotaxis of GFP-cotransfected Mo-DCs (uncoated filters; E). Error bars indicate \pm SD. ${ }^{*} P<.05$. Each experiment was repeated at least 3 times independently. Each single experiment was performed in duplicate.

Control cells and cytohesin-1 knockdown cells were differentially labeled with either CFSE or TAMRA dyes, and equal numbers of these cell groups were subsequently injected into the hind footpad of wild-type C57/BL6 mice. ${ }^{31}$ To exclude a perturbing effect of the dyes themselves on in vivo migration of DCs, the labeling of the respective populations was interchanged between experiments (Figure 4B,C). Twenty-four to 30 hours after injection, the draining (popliteal) lymph nodes (LNs) were extracted from these animals; single-cell suspensions were prepared and analyzed by FACS. Figure 4A shows that cytohesin-1 knockdown strongly reduced the migration of mature DCs to the draining lymph node in vivo. The numbers of BM-DCs that reached the $\mathrm{LN}$ were decreased by approximately $50 \%$ compared with control BM-DCs (set to $100 \%$ migration). Therefore, cytohesin-1 protein expression in DCs appears highly important for efficient migration of such cells from the tissue periphery to secondary lymphoid organs.

\section{Cytohesin-1 selectively regulates 3D migration of integrin-bearing DCs}

Using life cell imaging microscopy, we further studied the bulk migratory behavior of mature BM-DCs. Migration within tissues requires coordinated interactions with constituents of the extracellular matrix. ${ }^{32}$ With the help of $3 \mathrm{D}$ collagen type I gels as a structural scaffold, we modeled such complex conditions and 

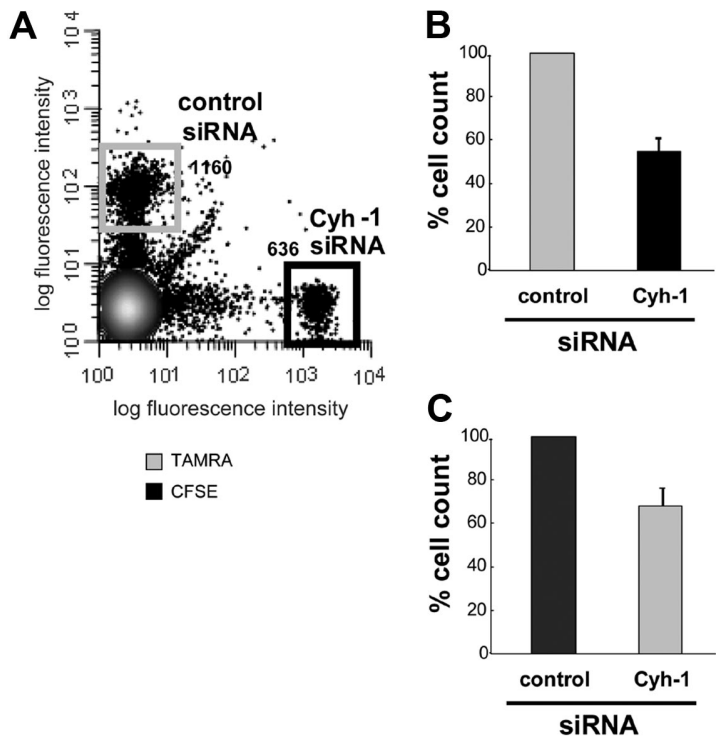

Figure 4. RNAi of cytohesin-1 strongly reduces in vivo migration of mature BM-DCs. Mature BM-DCs after transfection with control siRNA or cytohesin-1 siRNA (for silencing efficiency, see Western blot analysis of cytohesin-1 expression after RNAi of cytohesin-1, panel 1C) were labeled with $10 \mu \mathrm{M}$ TAMRA or $1 \mu \mathrm{M}$ CFSE, respectively. Cytohesin-1 knockdown DCs $\left(2.5 \times 10^{5}\right)$ and control DCs $\left(2.5 \times 10^{5}\right)$ were injected into the hind footpad of wild-type C57/BL6 mice. Flow cytometric analysis of cell suspensions from the extracted draining (popliteal) lymph nodes 24 to 30 hours after injection shows that in vivo migration of cytohesin-1 knockdown $B M-D C s$ is strongly reduced, compared with control cells $(A, B)$. To exclude fluorochrome effects on DC migration, the staining was switched between the experiments $(C)$. Control BM-DCs are set to $100 \%$ migration $(B, C)$. Error bars indicate \pm SD. Each experiment was repeated 3 times independently.

analyzed the migration of BM-DCs in these settings. Stimulation with CCL19 induced vigorous and directional migration of control DCs (Figure 5A,B and corresponding Videos S1-S4). In contrast, RNAi of cytohesin-1 strongly impaired CCL19-stimulated migration of BM-DCs within collagen (Figure 5C and corresponding Videos S5,S6). Using the ImageJ plug-in for manual tracking, we detected that average migratory speed (velocity), forward migration index, and directionality of cytohesin-1 knockdown DCs were significantly reduced compared with control cells (Figure 5E,F). Because Lämmermann et al had shown that the inhibition of the RhoA/myosin II pathway by blebbistatin resulted in the abrogation of DC contractility in $3 \mathrm{D}$ environments, ${ }^{25}$ we opted to use RNAi of RhoA in our assays. Strikingly, knockdown of RhoA yielded a highly similar phenotype as that of cytohesin-1 (Figure 5D-F and corresponding Videos S7,S8).

Lämmermann et $\mathrm{al}^{25}$ had shown that integrins are not required for BM-DC migration in 3D environments and in vivo. Using quadruple knockout BM-DCs, which do not express integrins on their surfaces, ${ }^{25}$ we therefore assessed whether cytohesin-1 was also required for integrin-independent migration of BM-DCs in 3D collagen. Figure 5 shows that 3D migration of wild-type cells was comparable with integrin-deficient BM-DCs, as expected (Figure $5 \mathrm{I}, \mathrm{K})$. Most interestingly, however, we found that RNAi of cytohesin-1, which was similarly efficient in both cell types (Figure $5 \mathrm{O}$ ), selectively affected 3D migration of integrin-bearing wildtype cells (Figure 5J,M,N). These findings suggest an important limiting function of cytohesin-1 in 3D leukocyte migration of integrin-positive DCs.

\section{Cytohesin-1 controls activation of RhoA in several cell types}

The apparent involvement of cytohesin- 1 in beta- 2 integrin activation and in integrin-independent interstitial migration of dendritic cells prompted us to investigate a possible and as-yet unappreciated upstream role of cytohesin-1 in RhoA activation of dendritic cells. Giagulli et al $^{15}$ had shown that RhoA is an important regulator of chemokine-induced T-cell adhesion to activated endothelium in vitro and in vivo, possibly by inducing or by stabilizing the high-affinity state of LFA-1. Rho GTPases are furthermore well known as key regulators of cell migration. In controlling diverse signal transduction pathways that link cell surface receptors to a variety of intracellular responses, they influence actin filament and microtubule dynamics, cell polarity, vesicular trafficking, and gene expression. ${ }^{33}$ Furthermore, Rho GTPases are essential in maintaining immune responses as they regulate lymphocyte and dendritic cell function and migration. ${ }^{34}$ Rho GTPases cycle between an active GTP-bound and an inactive GDP-bound state. The active GTP-loaded enzyme selectively interacts with downstream effector molecules. Activation of the GTPases was analyzed with the help of enzyme linked immunosorbent assays (ELISAs), using immobilized Rac or RhoA effector proteins PAK-1 and rhotekin, respectively. We observed that RNAi of cytohesin-1 reduces GTP loading of RhoA, whereas the activity of Rac is enhanced by cytohesin-1 knockdown in Mo-DCs (Figure 6A). Furthermore, a consistent reduction of RhoA activity is observed upon cytohesin-1 or RhoA protein knockdown (Figure 6B) in BM-DCs.

Significantly, the control of RhoA activity by cytohesin-1 is not restricted to immune cells. Overexpression of wild-type cytohesin-1 in HeLa cells results in an increase of RhoA activity, compared with the control sample that had been transfected with the vector. The GEF function of cytohesin- 1 is required for RhoA activation through this pathway, because overexpression of the cytohesin-1 E157K (GEF minus) mutant ${ }^{23}$ abrogates this effect (Figure $6 \mathrm{C}$ ). A role of cytohesin-1 function upstream of RhoA in this cell type was also confirmed by the alternative method of effector protein pull-down and subsequent Western blot analysis (Figure 6D). Consistently, Rac activity was significantly enhanced by cytohesin-1 RNAi in HeLa cells as well (Figure 6D).

To corroborate a full coherence of the functional activities of RhoA and cytohesin- 1 in all test systems used, we confirmed that RNAi of RhoA also reduces a CCL19-induced activation epitope expression of beta-2 integrins in Mo-DCs (Figure 6E). Furthermore, RhoA was found to be required for BM-DC adhesion to endothelioma cells under physiological flow conditions (Figure $6 \mathrm{~F})$. Finally, overexpression of RhoA enhances adhesion of MoDCs to ICAM-1-Fc, whereas the expression of the GTP-loadingdeficient mutant T19N abrogates this effect (Figure 6G).

We had previously shown that the functional activity of cytohesin-1 in human lymphocytes is regulated by PI3-kinasedependent membrane recruitment of the protein..$^{20}$ Consistently, we found that CCL19-dependent membrane recruitment of cytohesin-1, or LFA-1 activation epitope expression in Mo-DCs (Figure S10A,B), can be blocked using the PI3 kinase inhibitor LY-294002. In accordance with these data, inhibition of PI3-kinase in mature BM-DCs yielded a reduced RhoA activity (Figure S10C).

Taken together, we conclude that cytohesin-1 acts upstream of RhoA in directing integrin-dependent dendritic cell adhesion, their migration in 3D collagen gels, and likely their migratory behavior in inflamed tissues.

\section{Actin polymerization and the generation of membrane protrusions are not affected by cytohesin-1 in migrating DCs}

Cell migration highly depends on actin polymerization leading to membrane protrusions such as lamellipodia and filopodia, and is 
A

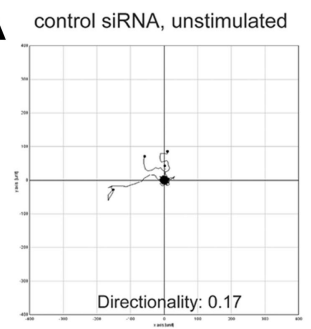

B

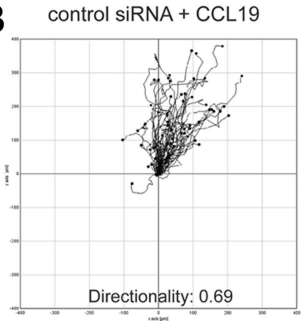

C

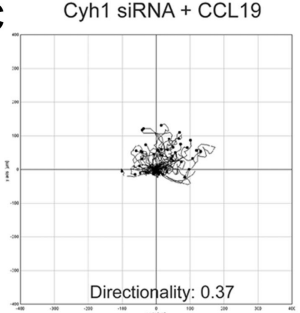

E

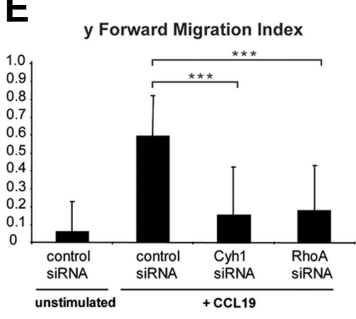

G

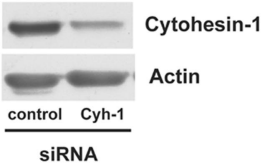

D

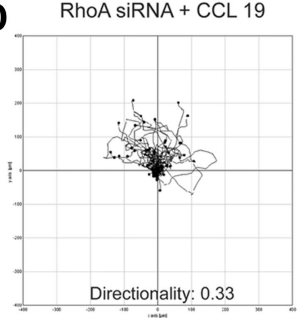

F

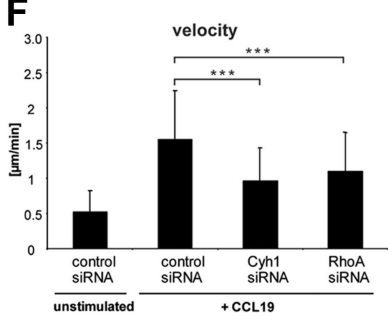

H

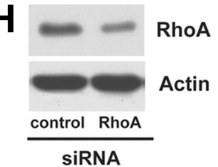

I

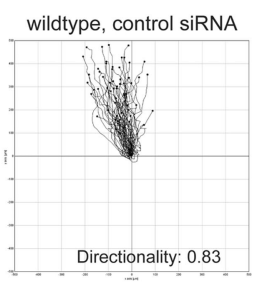

K

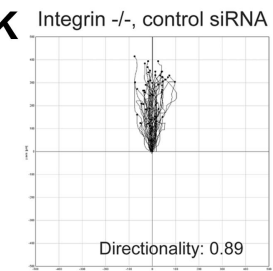

M

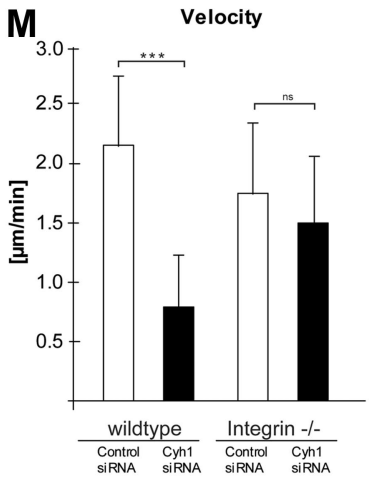

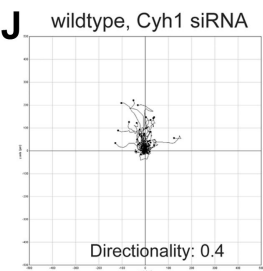

L Integrin -/-, Cyh1 siRNA

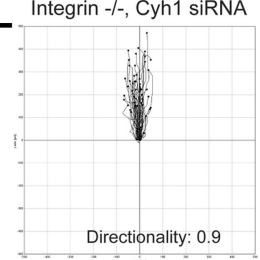

N y Forward Migration Index

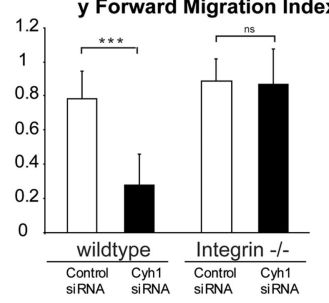

0

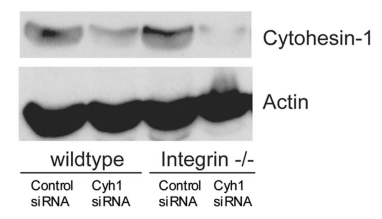

Figure 5. RNAi of cytohesin-1 strongly reduces chemotaxis of mature integrin(+/+) but not of integrin(-/-) BM-DCs in 3-dimensional collagen. Using 3D collagen type I gels, we analyzed the role of cytohesin-1 in BM-DC complex matrix migration. Quantification of time series after live cell imaging shows that directionality, y-forward migration index, and velocity of cytohesin-1 knockdown cells toward the chemokine CCL19 are strongly reduced (C,E,F). RNAi of RhoA results in a highly similar phenotype (D-F). Respective silencing efficiencies are shown by Western blot analysis (G,H). Quantification of 3D migration of integrin-deficient BM-DCs shows that directionality, $y$-forward migration index, and velocity of cytohesin-1 knockdown cells toward the chemokine CCL19 are strongly reduced in wild-type, that is, integrin(+/+) BM-DCs $(J, M, N)$ but not in integrin(-/-) BM-DCs (L-N). Efficiency of cytohesin-1 RNAi was comparable in both cell types, as shown by Western blot analysis (O). For every $3 \mathrm{D}$ migration experiment, the tracks of 60 individual cells were monitored over a period of 3 hours by capturing digital images every 5 minutes. Cell directionality, forward migration index, and velocity were calculated and visualized as plots (A-D, I-L) and animated plots (Videos S1-S8) by analyzing the acquired data with the Chemotaxis and Migration tool plug-in (Ibidi) for ImageJ. Error bars indicate \pm SD. ${ }^{\star \star \star} P<.001$; ns indicates not significant. The data are representative for 3 independent experiments.

regulated by tight coordination of Rho GTPase activity. ${ }^{35,36} \mathrm{Be}-$ cause cytohesin-1 knockdown strongly impairs DC migration and activation of RhoA, we further analyzed whether the actin network in migrating DCs is affected by cytohesin- 1 . To this end, DCs were fixed by formaldehyde within 3D collagen gels and stained with Cy3-conjugated phalloidin. Mature DCs transfected with control siRNA show F-actin at the cell cortex and within membrane protrusions (Figure 7A). Mature DCs lack stress fibers, which is known to be characteristic for leukocytes. ${ }^{37}$ The same, unaltered phenotype can be detected when cytohesin-1 expression in DCs is repressed by RNAi (Figure 7A). Thus, cytohesin-1 does apparently not affect polymerization and arrangement of actin fibers in membrane protrusions. Similarly, RNAi of RhoA does not influence the integrity of the peripheral actin network in mature DCs (Figure 7B), whereas, in contrast, RNAi of Rac1 completely inhibits generation of actin-based membrane protrusions (Figure 7C). These results are in good accordance with the recently published paper by Lämmermann et $\mathrm{al},{ }^{25}$ who showed that RhoA does not regulate the front of migrating DCs, but that the small GTPase instead controls contractility of the cell body, which is important for flexible cell shape changes during DC migration in tissue environments.

\section{Discussion}

In this study, we have described an important role of the guanine nucleotide exchange factor cytohesin- 1 in the regulation of cellular processes, as well as signaling pathways, which affect the regulation of adhesion and migration of human and murine dendritic cells. Overexpression analyses had implicated cytohesin-1 early on in the activation of the beta- 2 integrin LFA- 1 in T cells. ${ }^{19}$ We show here that cytohesin-1 expression in dendritic cells, which were derived from primary monocytes, is required for beta- 2 integrin adhesion under static and under dynamic flow conditions, as well as for chemokine-dependent conformational activation of this adhesion receptor. Furthermore, we discovered an entirely novel function of cytohesin-1 in controlling interstitial migration of integrin-bearing DCs, but not of integrin-depleted DCs. The signaling pathway discriminating between the 2 conditions has been identified as well: cytohesin-1 acts upstream of the GTPase RhoA, and this novel signaling axis is required for integrinmediated functions in the regulation of immune cell adhesion and migration. 

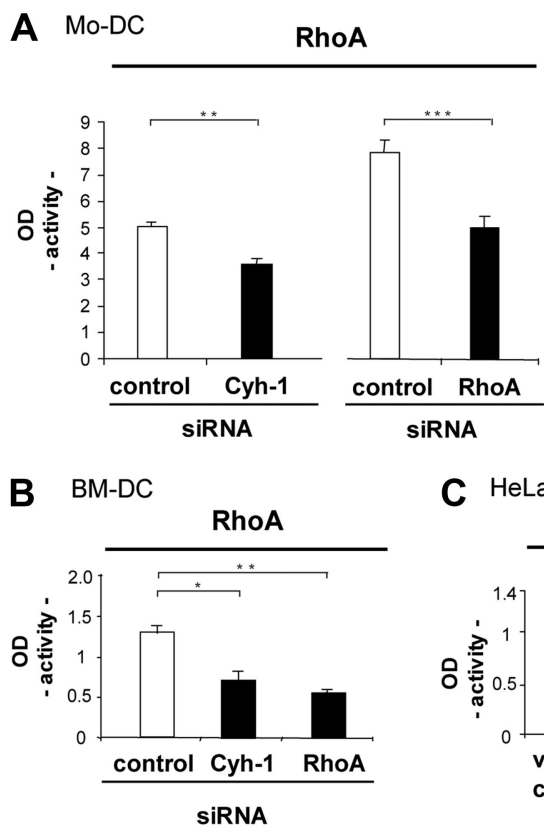

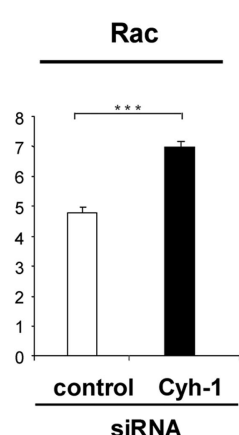

C HeLa

RhoA

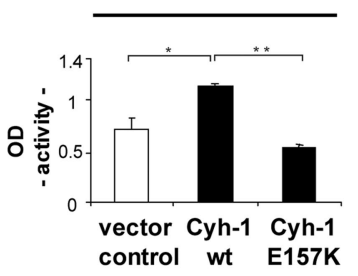

D
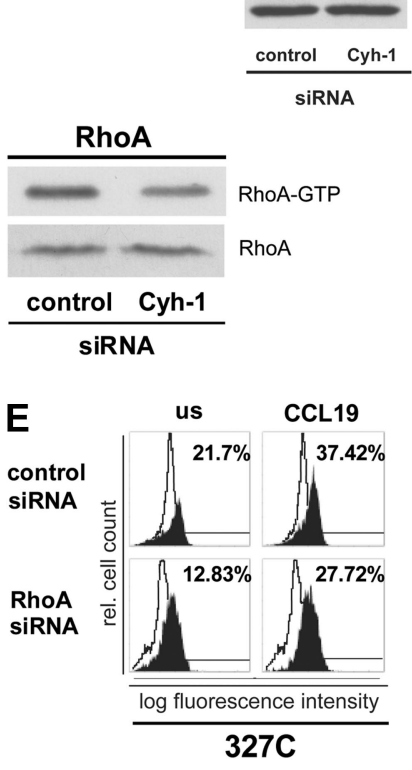

Cytohesin-1

Actin

.
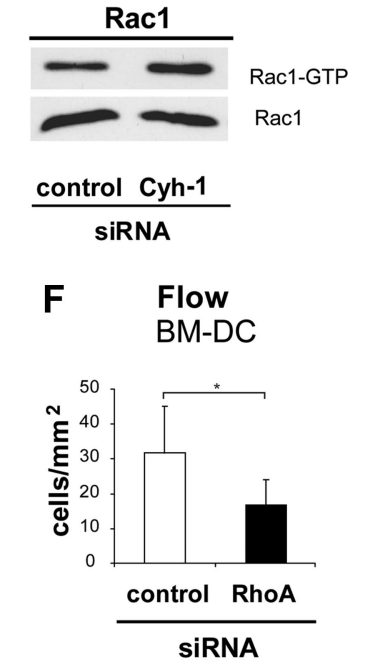

G

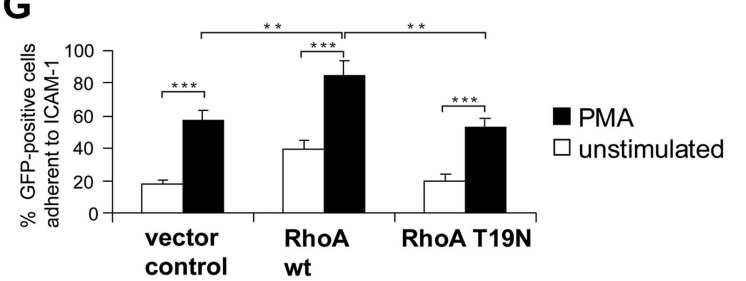

Figure 6. Cytohesin-1 controls the activity of RhoA in several cell types. RhoA is an important regulator of beta- 2 integrin activation epitope expression and adhesion of DCs. Using a commercial enzyme linked assay (ELISA), we could show that in Mo-DCs the activity (GTP loading) of the small GTPase RhoA is strongly reduced in cytohesin-1 knockdown cells, compared with control cells. As a positive control for the assay, RhoA activity of RhoA knockdown Mo-DCs was analyzed, which also shows the expected reduction of RhoA activity. The same system was used to measure Rac activity, where RNAi of cytohesin-1 results in an increase of GTP loading (A). Consistently, RhoA activity of RhoA knockdown BM-DCs was also found reduced (B). Overexpression of wild-type cytohesin-1 in EGF-stimulated (50 nM, 1 hour) HeLa cells results in an increase of RhoA activity, compared with the vector control. In contrast, overexpression of the cytohesin-1 E157K mutant results in reduced RhoA activity (C). Cytohesin-1 knockdown down-regulates RhoA activity, but up-regulates GTP loading of Rac1 in EGF-stimulated HeLa (50 nM; 1 hour) cells, as detected by effector protein pull-down and Western blot analysis (panel D bottom panels). Knockdown of RhoA specifically reduces expression of the CD18 327C epitope in Mo-DCs (E) or adhesion under flow of BM-DCs to brain endothelioma (B end5) cells (F). Overexpression of wild-type RhoA but not of the GTP-loading mutant T19N increases static adhesion of GFP-cotransfected Mo-DCs to ICAM-1-Fc (G). In the static adhesion assay, Mo-DCs were stimulated with $50 \mathrm{ng} / \mathrm{mL}$ PMA for 60 minutes at $37^{\circ} \mathrm{C}(\mathrm{G})$. Error bars indicate $\pm \mathrm{SD}$. ${ }^{\star \star *} P<.001,{ }^{\star \star} P<.01$, ${ }^{\star} P<.05$. Each experiment was repeated at least 3 times independently. Each single experiment was performed in duplicate.

Beta-2 integrin activation by chemokines is a complex process that involves the regulation of conformational equilibria of these proteins. ${ }^{28}$ At least 3 conformations have been reported: resting (none or very little ligand binding), and intermediate and high affinity for ligand. Dendritic cells had been reported earlier to be much more restricted in beta- 2 integrin activation than other leukocytes under certain conditions, but these studies had not used chemokine-dependent triggering or physiological flow conditions to test for integrin activation of DCs. Woolf et al ${ }^{38}$ had shown that immobilized chemokines and flow forces are important requirements for the activation of circulating as well as migrating leukocytes, even if these cells migrate in lymph nodes. Our novel data reveal that integrins of mature DCs are capable of adhering to activated endothelia under physiological flow conditions in a cytohesin-1-dependent fashion. Apparently, beta-2 integrins of mature human dendritic cells respond normally to such stimuli, which is in full accordance with the aforementioned study.

The precise sequence of events of chemokine-mediated integrin activation during physiological responses is not known, but forces imposed by the blood flow apparently play an important role as well as protein binding events at the cytoplasmic tails of integrins. Cytohesin-1 had been shown to bind to the membrane-proximal region of the beta-2 integrin cytoplasmic tail, and this is required for adhesion and activation epitope expression. Importantly, the ARF-GEF function is not required for integrin activation, but was found to be important for adhesion and spreading. ${ }^{23}$ RhoA is a small GTPase that links integrin signaling to actin cytoskeletal rearrangements in many cell types. By use of blocking peptides, Giagulli et $\mathrm{l}^{15}$ had found that RhoA is required for leukocyte beta- 2 integrin activation in endothelial arrest. These findings were recently confirmed and extended by Pasvolsky et al, ${ }^{39}$ who showed that RhoA blocking peptides regulate intermediate affinity epitope expression of LFA-1 in $\mathrm{T}$ cells in flow. We now show that cytohesin-1 regulates the GTP loading of RhoA in leukocytes and we consistently report that both cytohesin-1 and RhoA mediate chemokine-induced activation epitope expression of CD18 in dendritic cells (Figure 2, Figure 6E, and data not shown). This places cytohesin-1 upstream of RhoA in beta-2 integrin activation. It is important to note here that this does not rule at all against an additional direct role of cytohesin-1/CD18 interactions in LFA-1 activation. ${ }^{23}$ Taken together, our novel data reveal a signaling link between cytohesin-1 and RhoA in beta- 2 integrin activation and adhesion of dendritic cells, which likely reflects a dual role of cytohesin- 1 in integrin binding and in downstream signaling to RhoA. 
A

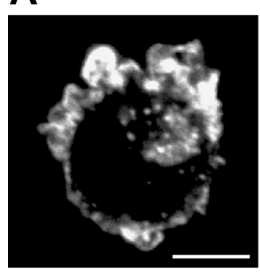

control

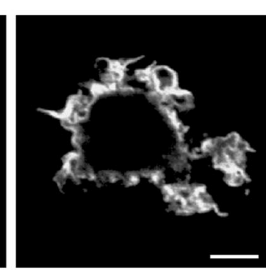

Cytohesin-1

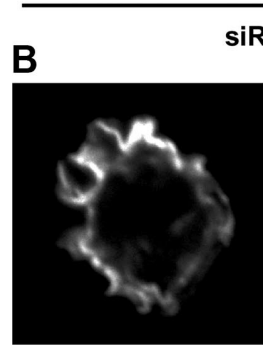

control

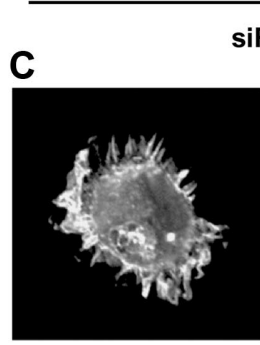

control

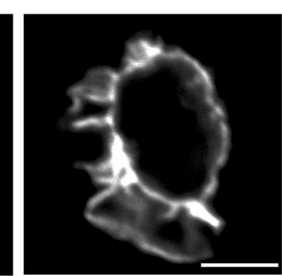

RhoA

SiRNA

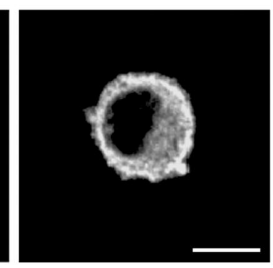

Rac1 cells with lamellipodia

cells without lamellipodia

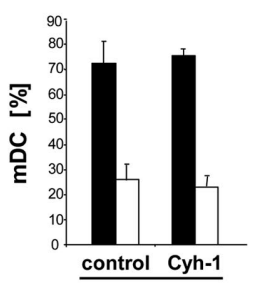

SIRNA
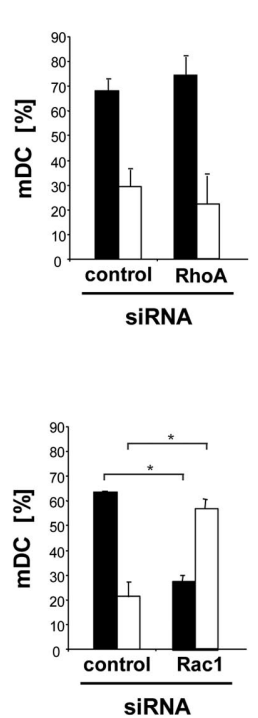

SiRNA

Figure 7. Cytohesin-1 is not involved in the formation of membrane protrusions. Microscopic visualization of F-actin by the use of Cy3-phalloidin shows that the formation of membrane protrusions in mature Mo-DCs is not influenced by RNAi of cytohesin-1, compared with control cells (A). RNAi of RhoA also shows the same unaltered phenotype $(B)$, whereas RNAi of Rac1 results in strong deficiencies in the formation of membrane protrusions (C). Quantification of membrane protrusions shows that after RNAi of cytohesin-1 respective RhoA $70 \%$ of all mature Mo-DCs have lamellipodia and do not differ from control cells $(A, B)$. In contrast, RNAi of Rac reduces formation of lamellipodia approximately $50 \%$ (C). Bars represent $5 \mu \mathrm{m}$. In each individual experiment, 200 mature DCs were counted. Each single experiment was performed in duplicate. Error bars indicate $\pm \mathrm{SD}$. ${ }^{*} P<.05$. Each experiment was repeated at least 3 times independently.

We show further and for the first time that cytohesin- 1 is required for migration of BM-DCs to lymph nodes in vivo and for directional migration of BM-DCs in $3 \mathrm{D}$ collagen gels in vitro. RhoA had been implicated in both functions. In fact, RNAi of RhoA or cytohesin-1 resulted in a highly similar phenotype in interstitial migration of DCs in our assay systems. Specific treatment of BM-DCs with RhoA or cytohesin-1 siRNA yielded a strong abrogation of chemokine-gradient direction sensing and migratory persistence (track length). Most strikingly, however, our analyses show that 3D migration of leukocytes in the presence or absence of integrins is phenotypically similar but not identical. Whereas integrin-positive cells specifically require the cytohesin-1/ RhoA signaling axis, integrin-null DCs require RhoA, ${ }^{25}$ but not cytohesin-1 (shown in this study) for 3D motility. It thus becomes apparent that leukocyte migration in $3 \mathrm{D}$ environments may also be subclassified into different types or "modes," reminiscent of what has been described for certain tumor cells. ${ }^{40,41}$ The fact that cytohesin-1 dependency discriminates between leukocytes migrating in the absence and presence of integrins raises the question how

the Rho pathway is regulated when both integrins and cytohesin-1 are absent and why this alternative pathway is not used when only cytohesin-1 is missing? One likely possibility is that in the absence of integrins, DCs use another migratory mode that does not depend on cytohesin as a Rho activator. This would imply that DCs can use phenotypically identical but molecularly distinct migratory strategies and suggest that there is more plasticity within the ameboid migration mode than expected. In these experiments, we have used cells that lack all surface integrins to elucidate a principal role of cytohesin-1 in 3D immune cell migration. Consistent with all of our earlier findings, we furthermore show a strong functional association of this protein with beta-2 integrins. However, these data do not exclude a possible cooperation of cytohesin-1 with other integrins in these functions.

How are cytohesin-1 functions linked to RhoA signaling? Cytohesin-1 is a guanine nucleotide exchange factor for ARFGTPases, and GDP/GTP exchange activities for other GTPases have never been reported. It is important to note here that a large body of work implicates cytohesin-2 (or ARNO) and -3 (Grp1) in ARF6 signaling upstream of Rac- $1 .^{42}$ Cytohesin-1 has never been implicated in direct activation of the endosomal and cytoskeletal signaling GTPase ARF6. In fact, data from our laboratory rule against such a link in T cells. ${ }^{43}$ RhoA and Rac activity are tightly coordinated in cell migration and are often controlled in a reciprocal fashion. ${ }^{44-46}$ Interestingly, we observed not only a down-regulation of RhoA activity by cytohesin-1 knockdown but an up-regulation of Rac activity as well. This indicates that the control of Rho GTPase activities by cytohesins in immune and nonimmune cells might be aspects of a common theme, involving specific roles of cytohesin- 1 in the RhoA pathway and of cytohesin$2 / 3$ in Rac signaling.

\section{Acknowledgments}

We thank Reinhard Fässler (MPI for Biochemistry, Martinsried, Germany) for providing quadruple integrin knockout mice, Percy Knolle (IMMEI, University of Bonn, Bonn, Germany) for providing the OT-1 mice, Don Staunton (ICOS, Seattle, WA) for providing antibody 327C, and Elisabeth Kremmer (Helmholtz Center Munich, Munich, Germany) for continuous supply with anti-cytohesin-1 antibodies. In addition, we thank members of the laboratory for discussion and advice.

This work was supported by the Deutsche Forschungsgemeinschaft (SFBs 654 and 704) and by grant no. 105845 of the Deutsche Krebshilfe.

\section{Authorship}

Contribution: T.Q., B.T., C.S., J.G., N.C., K.D., and T.L. carried out the experiments; R.F., R.A., C.W., and M.S. contributed to research design and data analysis; J.G. and L.F. established study protocols; and W.K. designed the study, wrote the paper, and acquired research funding.

Conflict-of-interest disclosure: The authors declare no competing financial interests.

Correspondence: Waldemar Kolanus, Life and Medical Sciences Institute, Laboratory for Molecular Immunology, University of Bonn, Karlrobert-Kreitenstr 13, 53115 Bonn, Germany; e-mail: wkolanus@uni-bonn.de. 


\section{References}

1. Hynes RO. Integrins: bidirectional, allosteric sig naling machines. Cell. 2002;110:673-687.

2. Bouschet T, Martin S, Kanamarlapudi V, Mundell S, Henley JM. The calcium-sensing receptor changes cell shape via a beta-arrestin-1 ARNO ARF6 ELMO protein network. J Cell Sci. 2007; 120:2489-2497.

3. Kim M, Carman CV, Springer TA. Bidirectional transmembrane signaling by cytoplasmic domain separation in integrins. Science. 2003;301:1720 1725.

4. Shamri R, Grabovsky V, Gauguet JM, et al. Lymphocyte arrest requires instantaneous induction of an extended LFA-1 conformation mediated by endothelium-bound chemokines. Nat Immunol. 2005;6:497-506.

5. Tadokoro S, Shattil SJ, Eto K, et al. Talin binding to integrin beta tails: a final common step in integrin activation. Science. 2003;302:103-106.

6. Calderwood DA. Talin controls integrin activation. Biochem Soc Trans. 2004;32:434-437.

7. Katagiri K, Hattori M, Minato N, Irie S, Takatsu K, Kinashi T. Rap1 is a potent activation signal for leukocyte function-associated antigen 1 distinct from protein kinase $\mathrm{C}$ and phosphatidylinositol3-OH kinase. Mol Cell Biol. 2000;20:1956-1969.

8. Reedquist KA, Ross E, Koop EA, et al. The small GTPase, Rap1, mediates CD31-induced integrin adhesion. J Cell Biol. 2000;148:1151-1158.

9. Lafuente EM, van Puijenbroek AA, Krause M, et al. RIAM, an Ena/VASP and Profilin ligand, interacts with Rap1-GTP and mediates Rap1-induced adhesion. Dev Cell. 2004;7:585-595.

10. Katagiri K, Maeda A, Shimonaka M, Kinashi T. RAPL, a Rap1-binding molecule that mediates Rap1-induced adhesion through spatial regulation of LFA-1. Nat Immunol. 2003:4:741-748.

11. Ménasché G, Kliche S, Bezman N, Schraven B Regulation of T-cell antigen receptor-mediated inside-out signaling by cytosolic adapter proteins and Rap1 effector molecules. Immunol Rev. 2007:218:82-91.

12. Heuer K, Sylvester M, Kliche S, et al. Lipid-binding $\mathrm{hSH} 3$ domains in immune cell adapter proteins. J Mol Biol. 2006;361:94-104.

13. Griffiths EK, Krawczyk C, Kong YY, et al. Positive regulation of $T$ cell activation and integrin adhesion by the adapter Fyb/Slap. Science. 2001;293: 2260-2263.

14. Peterson EJ, Woods ML, Dmowski SA, et al. Coupling of the TCR to integrin activation by Slap-130/Fyb. Science. 2001;293:2263-2265.

15. Giagulli C, Scarpini E, Ottoboni L, et al. RhoA and zeta PKC control distinct modalities of LFA-1 activation by chemokines: critical role of LFA- 1 affinity triggering in lymphocyte in vivo homing. Immunity. 2004;20:25-35

16. Weber KS, Klickstein LB, Weber PC, Weber C. Chemokine-induced monocyte transmigration requires cdc42-mediated cytoskeletal changes. Eur J Immunol. 1998;28:2245-2251.

17. Montanez E, Ussar S, Schifferer M, et al.
Kindlin-2 controls bidirectional signaling of integrins. Genes Dev. 2008;22:1325-1330.

18. Moser M, Nieswandt B, Ussar S, Pozgajova M, Fässler R. Kindlin-3 is essential for integrin activation and platelet aggregation. Nat Med. 2008; 14:325-330.

19. Kolanus W, Nagel W, Schiller B, et al. Alpha L beta 2 integrin/LFA-1 binding to ICAM-1 induced by cytohesin-1, a cytoplasmic regulatory molecule. Cell. 1996:86:233-242.

20. Nagel W, Zeitlmann L, Schilcher P, Geiger C Kolanus J, Kolanus W. Phosphoinositide 3-OH kinase activates the beta2 integrin adhesion pathway and induces membrane recruitment of cytohesin-1. J Biol Chem. 1998;273:1485314861

21. Nagel W, Schilcher P, Zeitlmann L, Kolanus W. The $\mathrm{PH}$ domain and the polybasic $\mathrm{c}$ domain of cytohesin-1 cooperate specifically in plasma membrane association and cellular function. Mo Biol Cell. 1998:9:1981-1994.

22. Weber KS, Weber C, Ostermann G, Dierks H, Nagel W, Kolanus W. Cytohesin-1 is a dynamic regulator of distinct LFA-1 functions in leukocyte arrest and transmigration triggered by chemokines. Curr Biol. 2001:11:1969-1974.

23. Geiger C, Nagel W, Boehm T, et al. Cytohesin-1 regulates beta-2 integrin-mediated adhesion through both ARF-GEF function and interaction with LFA-1. EMBO J. 2000;19:2525-2536.

24. Friedl $P$, Entschladen F, Conrad C, Niggemann B, Zanker KS. CD4+ T lymphocytes migrating in three-dimensional collagen lattices lack focal adhesions and utilize beta1 integrin-independent strategies for polarization, interaction with collagen fibers and locomotion. Eur J Immunol. 1998; 28:2331-2343.

25. Lämmermann T, Bader BL, Monkley SJ, et al. Rapid leukocyte migration by integrinindependent flowing and squeezing. Nature. 2008;453:51-55

26. Boehm T, Hofer S, Winklehner P, et al. Attenuation of cell adhesion in lymphocytes is regulated by CYTIP, a protein which mediates signal complex sequestration. EMBO J. 2003;22:1014-1024.

27. Elbashir SM, Harborth J, Weber K, Tuschl T. Analysis of gene function in somatic mammalian cells using small interfering RNAs. Methods. 2002;26: 199-213.

28. Constantin G, Majeed M, Giagulli C, et al. Chemokines trigger immediate beta2 integrin affinity and mobility changes: differential regulation and roles in lymphocyte arrest under flow. Immunity. 2000;13:759-769.

29. Beals CR, Edwards AC, Gottschalk RJ, Kuijpers TW, Staunton DE. CD18 activation epitopes induced by leukocyte activation. J Immunol. 2001; 167:6113-6122.

30. Kim M, Carman CV, Yang W, Salas A, Springe TA. The primacy of affinity over clustering in regulation of adhesiveness of the integrin \{alpha\}L\{beta\}2. J Cell Biol. 2004;167:1241-1253.

31. Worbs T, Mempel TR, Bolter J, von Andrian UH, Forster R. CCR7 ligands stimulate the intranodal motility of T lymphocytes in vivo. J Exp Med 2007;204:489-495.

32. Friedl P, Brocker EB, Zanker KS. Integrins, cell matrix interactions and cell migration strategies: fundamental differences in leukocytes and tumo cells. Cell Adhes Commun. 1998;6:225-236.

33. Raftopoulou M, Hall A. Cell migration: Rho GTPases lead the way. Dev Biol. 2004;265:23-32.

34. Scheele JS, Marks RE, Boss GR. Signaling by small GTPases in the immune system. Immunol Rev. 2007;218:92-101.

35. Antoshechkin I, Han M. The C. elegans evl-20 gene is a homolog of the small GTPase ARL2 and regulates cytoskeleton dynamics during cytokinesis and morphogenesis. Dev Cell. 2002;2: 579-591.

36. Ridley AJ. Rho GTPases and actin dynamics in membrane protrusions and vesicle trafficking Trends Cell Biol. 2006;16:522-529.

37. Friedl P, Borgmann S, Brocker EB. Amoeboid leukocyte crawling through extracellular matrix: lessons from the Dictyostelium paradigm of cell movement. J Leukoc Biol. 2001;70:491-509.

38. Woolf E, Grigorova I, Sagiv A, et al. Lymph node chemokines promote sustained T lymphocyte motility without triggering stable integrin adhesiveness in the absence of shear forces. Nat Immunol. 2007;8:1076-1085.

39. Pasvolsky R, Grabovsky V, Giagulli C, et al. RhoA is involved in LFA-1 extension triggered by CXCL12 but not in a novel outside-in LFA-1 activation facilitated by CXCL9. J Immunol. 2008; 180:2815-2823.

40. Sanz-Moreno V, Gadea G, Ahn J, et al. Rac activation and inactivation control plasticity of tumor cell movement. Cell. 2008;135:510-523.

41. Sahai E, Marshall CJ. Differing modes of tumour cell invasion have distinct requirements for $\mathrm{Rho/}$ ROCK signalling and extracellular proteolysis. Nat Cell Biol. 2003;5:711-719.

42. Myers KR, Casanova JE. Regulation of actin cy toskeleton dynamics by Arf-family GTPases. Trends Cell Biol. 2008;18:184-192.

43. Knorr T, Nagel W, Kolanus W. Phosphoinositides determine specificity of the guanine-nucleotide exchange activity of cytohesin-1 for ADPribosylation factors derived from a mammalian expression system. Eur J Biochem. 2000;267: 3784-3791.

44. Zondag GC, Evers EE, ten Klooster JP, Janssen L, van der Kammen RA, Collard JG. Oncogenic Ras downregulates Rac activity, which leads to increased Rho activity and epithelialmesenchymal transition. J Cell Biol. 2000;149: 775-782.

45. Williams MJ, Habayeb MS, Hultmark D. Reciprocal regulation of Rac1 and Rho1 in Drosophila circulating immune surveillance cells. J Cell Sci. 2007:120:502-511.

46. Herbrand U, Ahmadian MR. p190-RhoGAP as an integral component of the Tiam1/Rac1-induced downregulation of Rho. Biol Chem. 2006;387 311-317. 